\title{
The Crucial Role of Bilateral Infraclavicular Nerve Blocks in the Anesthetic Management of a Trauma Patient
}

\author{
Eric P. Chiang ${ }^{1}$, Paul Dangerfield ${ }^{1}$, Jaideep H. Mehta ${ }^{2}$, Marian Sherman ${ }^{1}$, Jeffrey S. Berger ${ }^{1}$ \\ ${ }^{1}$ Department of Anesthesiology and Critical Care Medicine, George Washington University Medical Center, Washington DC, USA; \\ ${ }^{2}$ Department of Anesthesiology, University of Texas Health Science Center, Houston, USA. \\ Email: Epchiang@email.gwu.edu
}

Received July $2^{\text {nd }}$, 2012; revised August $3^{\text {rd }}$, 2012; accepted August 25 $5^{\text {th }}, 2012$

\begin{abstract}
Bilateral brachial plexus blocks and regional anesthesia in trauma patients are rarely performed due to potential complications when using these techniques. We illustrate a case in which bilateral infraclavicular nerve blocks were placed as part of a multimodal approach to pain management in a trauma patient. We discuss potential hazards, important considerations, and rationale for attempting this procedure. Ultimately, performing bilateral brachial plexus nerve blocks in trauma patients is a viable option when choosing pain management techniques.
\end{abstract}

Keywords: Bilateral Brachial Plexus Blocks; Regional Anesthesia in Trauma; Pain Management in Trauma

\section{Introduction}

In spite of the recent advances in regional anesthesia, there are still concerns for systemic toxicity when performing blocks at multiple sites [1]. Particularly, bilateral brachial plexus blocks are not often performed because of the increased concern for technique-related complications, and few clinical scenarios warrant their use [2]. The field of trauma is one arena where performing bilateral procedures may be clinically indicated [3]. The trauma situation, however, may raise other concerns for regional anesthesia and, therefore, its utility is often overlooked. We present a case where multimodal analgesia including bilateral infraclavicular nerve blocks was integral to the anesthetic management of a patient with multisystem trauma.

\section{Case Report}

We report the case of a 36-year-old woman who was admitted to the emergency department after sustaining multiple injuries from a three story fall.

The initial trauma work up showed bilateral maxillary fractures, left frontal subarachnoid hemorrhage (SAH), pneumocephalus, and multiple injuries to her extremities. The extremity injuries included: minimally displaced right acetabular fracture, right medial femoral epicondyle impaction fracture, left displaced open radius and ulna fracture, carpal separation, left carpal fracture and elbow dislocation, bilateral open knee, right ulnar fracture, and right toe dislocation. Notably, there were no intra-tho- racic or intra-abdominal injuries.

After stabilization in the intensive care unit (ICU), the patient was brought to the operating room. General anesthesia with multiple large bore intravenous access and intracranial pressure (ICP) monitoring were employed to manage the patient while the orthopedic team completed an incision and drainage of the bilateral lower extremities, closed reduction of the left upper extremity, and pinning of the right wrist. At this point, ICP monitors registered a rise from $15 \mathrm{~mm} \mathrm{Hg}$ to $30 \mathrm{~mm} \mathrm{Hg}$. The patient's ICP responded to mannitol and dexamethasone and the orthopedic injuries were temporized. No further orthopedic procedures were attempted at this time; and an urgent computerized axial tomography scan of her head ruled out an expanding pneumocephalus or SAH. The patient was allowed to stabilize overnight in the ICU; she remained intubated and sedated in anticipation of returning to the operating the following day.

The patient needed to return to the operating room for simultaneous bilateral reduction and fixation of her upper extremity fractures. Collaborating teams felt that pain and sympathetic stimulation were factors that contributed to the sudden rise in ICP [4]. Ultrasound guided bilateral continuous infraclavicular nerve blocks were planned for pain control and sympathetic stimulation management.

The possibility of regional anesthesia was discussed with the patient's family because the patient was intubated and sedated medications. Although the patient was sedated, she was still able to follow commands and a neurologic exam of her upper extremities revealed bilat- 
eral ulnar deficits, the left worse than the right, and the findings were documented prior to the start of the procedures.

The patient was medicated with $2 \mathrm{mg}$ midazolam and 50 ug fentanyl. The block anatomy was not obscured by the cervical collar or the subclavian line and both sides were prepared with Betadine and draped with sterile towels. The relevant anatomy was identified on a Sonosite M-Turbo ultrasound machine (Sonosite, Bothell, WA) using a 5- to $10-\mathrm{MHz}$ curvilinear probe (Sonosite, Bothell, WA). The posterior, medial, and lateral cords were imaged (Figure 1) and a 4-inch, 18-guage Touhy needle (BBraun, Bethlehem, PA) which was inserted using an in-plane approach until the tip of the needle was adjacent to the cords and $2 \mathrm{ml}$ of local anesthetic was injected to confirm the location. The block was performed with $20 \mathrm{ml}$ of ropivicaine $0.5 \%$ with epinephrine 1:200,000. The local anesthetic was injected slowly and aspirations was performed after every $5 \mathrm{ml}$. A 20-guage Perifix catheter (BBraun, Bethlehem, PA) was threaded and the location confirmed by injecting $2 \mathrm{ml}$ of air through the catheter (Figure 2). The procedure was then repeated on the opposite side using the same technique. A continuous infusion of ropivicaine $0.2 \%$ was started at 4 $\mathrm{ml} / \mathrm{h}$ on each side.

After this procedure, the patient returned to the operating room to undergo definitive upper extremity repair. With the benefit of the continuous infraclavicular nerve blocks, the patient required only minimal sedation as delivered by low dose propofol $75 \mathrm{mcg} / \mathrm{kg} / \mathrm{m}$ and fentanyl $2 \mathrm{mcg} / \mathrm{kg} / \mathrm{h}$ infusions unchanged from the ICU settings. The patient tolerated the seven hour procedure and did not experience another increase in ICP intraoperatively.

The patient continued to have excellent pain control post-operatively and required minimal fentanyl and versed for sedation while undergoing a magnetic resonance imaging scan later that night. The patient required no medications beyond the local anesthetic infusions, the sedation was tapered off, and the patient was extubated the next day in the ICU. One additional non-sedating medication the patient received was intravenous acetaminophen as part of her multimodal pain regimen. The catheters were evaluated daily for 7 days and provided excellent pain relief as her mental status and neurologic deficits gradually improved. Upon removal, the catheters were intact, the site was non-tender and non-erythematic, and no complications were noted. The patient continued to do well and was discharged to the Acute Rehab Unit on postoperative day eleven.

\section{Discussion}

Bilateral brachial plexus blocks are rarely performed be- cause few clinical situations necessitate bilateral procedures. There are also increased concerns for local anesthetic toxicity, phrenic nerve paralysis, and pneumothroax with bilateral blocks. The trauma patient may offer a scenario where simultaneous bilateral procedures may be clinically indicated. But pain management and regional anesthesia is often difficult in the setting of traumatic injuries. There are often limitations on analgesic options in the prehospital setting, and even when the patient arrives in the emergency department, pain is often undertreated [5,6]. Although pain management has been shown to reduce morbidity and improve long-term outcomes in trauma patients, there is fear that addressing the pain may mask the physical or neurologic exams [7].

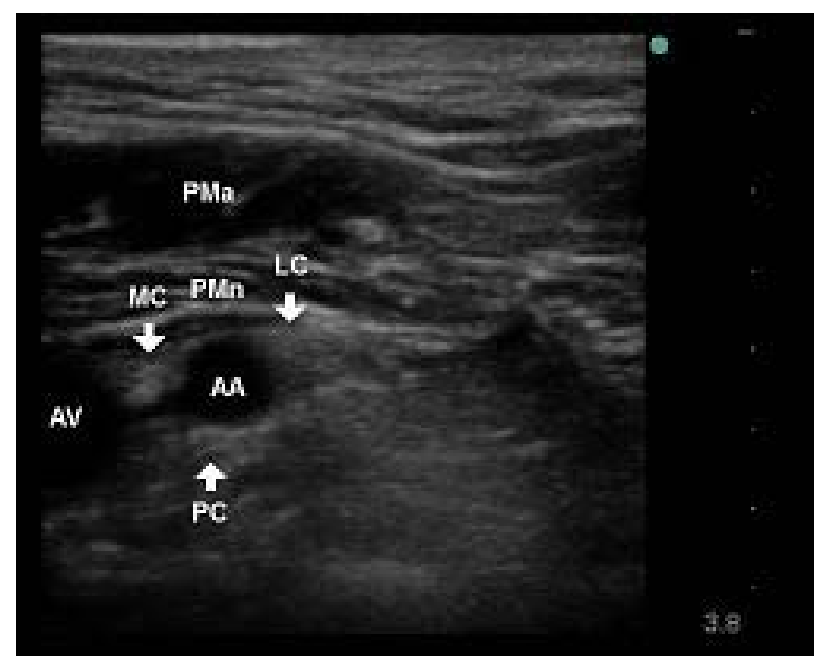

Figure 1. Sonogram of the infraclavicular brachial plexus. AA indicates axillary artery; AV, axillary vein; PC, posterior cord; MC, medial cord; LC, laterial cord; PMA, pectoralis major; PMn, pectoralis minor.

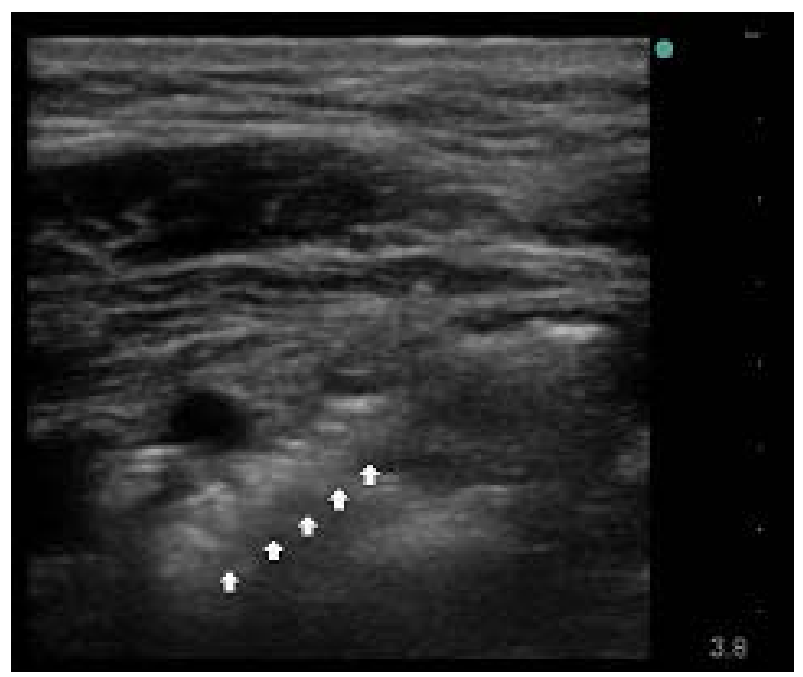

Figure 2. Catheter location is confirmed with injection of 2 $\mathrm{ml}$ of air (arrows). 
In the trauma setting, physicians are concerned that regional anesthesia may mask neurologic deficits and compartment syndrome. A recent review article by Mar et al. concluded that there is no evidence that regional anesthesia delays the diagnosis of compartment syndrome in patients who are adequately monitored [8]. In fact, decreasing the amount of narcotics and other sedating medications was significant because it eliminated variables and allowed the teams to more clearly evaluate the patient's mental status.

In dealing with existing neurologic deficits, it is important to inform the patient of the risk of neurological complications and document the pre-block neurologic exam in the patient chart. Additional efforts to help reduce neurologic complications are to avoid paresthesia techniques while placing the block, careful patient positioning during the surgical procedure, and avoiding constrictive dressings and casts postoperatively [9]. We adhered to all of these neurologic safety precautions in our patient. The American Society of Regional Anesthesia and Pain Medicine (ASRA) Practice Advisory stated that they can neither confirm nor refute increased adverse effects with regional anesthesia in patients with pre-existing neurologic deficits, and that if an increased risk is present, it is likely of minimal magnitude. Also patients may develop new deficits independent of anesthetic choice [10].

The incidence of seizures from local anesthetic toxicity after a peripheral nerve block is approximately $0.01 \%$ to $0.2 \%$ and the number is still decreasing [3]. In the past with nerve stimulator techniques, large doses of longacting local anesthetics were administered as a single bolus and the volume was an important determinant of the success of brachial plexus block. Recently, however, ultrasound-guided techniques allow for local anesthetic to be administered more accurately with lower doses of the initial medication bolus [1]. The maximum recommended dose of local anesthetic depends on the type of local anesthetic used, the site of local anesthetic injection, and patient-related factors (age, organ dysfunction, and pregnancy) that may alter the pharmacokinetics of the local anesthetic. We used $20 \mathrm{ml}$ of Ropivacaine $0.5 \%$ on each side which calculates to a total of $200 \mathrm{mg}$ of Ropivacaine. This is much lower than the $300 \mathrm{mg}$ of Ropivacaine officially recommended as the maximum dose for brachial plexus blocks [11]. Continuous nerve catheters allow for titration of the doses and helps extends the duration of the block. The bilateral blocks were an adjuvant and not the sole technique, thus a lower infusion rate was possible.

The incidence of phrenic nerve block is reduced with ultrasound guidance and lower doses of local anesthetic. In the past, bilateral interscalene blocks were contraindicated because total paresis of the diaphragm could result in respiratory insufficiency [12]. The incidence of phrenic nerve block is lower in supraclavicular blocks and there have been successful bilateral infraclavicular blocks without phrenic nerve block or deterioration of respiretory function [3].

The incidence of reported pneumothroax with brachial plexus blocks has decreased because when placing the infraclavicular block under ultrasound guidance, the lung field may be visualized, avoiding puncture. In a retrospective analysis of over one thousand patients who received an infraclavicular block under ultrasound guidance, Sandu et al. reported no cases of nerve injury, pneumothorax, or local anesthetic toxicity [13].

In conclusion, advances in ultrasound guidance and continuous nerve catheter techniques have helped reduce the complications associated with bilateral brachial plexus blocks. Bilateral infraclavicular nerve blocks may play a crucial role for pain management in the trauma setting when simultaneous bilateral procedures are necessary.

\section{REFERENCES}

[1] N. S. Sandhu, B. Maharlouei, B. Patel, E. Erkulwater and P. Medabalmi, "Simultaneous Bilateral Infraclavicular Brachial Plexus Blocks with Low-Dose Lidocaine Using Ultrasound Guidance,” Anesthesiology, Vol. 104, No. 1, 2006, pp. 199-201. doi:10.1097/00000542-200601000-00028

[2] C. D. France, Z. Salahuddin and A. Rafizad, "Bilateral Brachial Plexus Block,” Anesthesia \& Analgesia, Vol. 98, No. 2, 2004, pp. 518-520. doi:10.1213/01.ANE.0000097441.67236.33

[3] J. Holborow and G. Hocking, "Regional Anaesthesia for Bilateral Upper Limb Surgery: A Review of Challenges and Solutions," Anaesthesia and Intensive Care, Vol. 38, No. 2, 2010, pp. 250-258.

[4] L. Rangel-Castillo, S. Gopinath and C. S. Robertson, "Management of Intracranial Hypertension," Neurologic Clinics, Vol. 26, No. 2, 2008, pp. 521-541. doi:10.1016/j.ncl.2008.02.003

[5] M. L. Borland, I. Jacobs and I. R. Rogers, "Options in Prehospital Analgesia,” Emergency Medicine, Vol. 14, No. 1, 2002, pp. 77-84. doi:10.1046/j.1442-2026.2002.00288.x

[6] J. J. Bazarian, J. McClung, Y. T. Cheng, W. Flesher and S. M. Schneider, "Emergency Department Management of Mild Traumatic Brain Injury in the USA,” Emergency Medicine, Vol. 22, No. 7, 2005, pp. 473-477. doi:10.1136/emj.2004.019273

[7] S. P. Cohen, P. J. Christo and L. Moroz, "Pain Management in Trauma Patients," American Journal of Physical Medicine \& Rehabilitation, Vol. 83, No. 2, 2004, pp. 142 161. doi:10.1097/01.PHM.0000107499.24698.CA

[8] G. J. Mar, M. J. Barrington and B. R. McGuirk, “Acute Compartment Syndrome of the Lower Limb and the Effect of Postoperative Analgesia on Diagnosis," British Journal of Anaesthesia, Vol. 102, No. 1, 2009, pp. 3-11. 


\section{doi:10.1093/bja/aen330}

[9] P. Bajaj, "Regional Anaesthesia in the Patient with PreExisting Neurological Dysfunction,” Indian Journal of Anaesthesia, Vol. 53, No. 2, 2009, pp. 135-138.

[10] J. M. Neal, C. M. Bernards, A. Hadzic, J. R. Hebl and Q. H. Hogan, "ASRA Practice Advisory on Neurologic Complications in Regional Anesthesia and Pain Medicine," Regional Anesthesia and Pain Medicine, Vol. 33, No. 5, 2008, pp. 404-415.

[11] P. H. Rosenberg, B. T. Veering and W. F. Urmey, "Maximum Recommended Doses of Local Anesthetics: A Multifactorial Concept," Regional Anesthesia and Pain Medicine, Vol. 29, No. 6, 2004, pp. 564-574. doi:10.1016/j.rapm.2004.08.003

[12] K. Maurer, G. Ekatodramis, J. Hodler, K. Rentsch, H. Perschak and A. Borgeat, "Bilateral Continuous Interscalene Block of Brachial Plexus for Analgesia after Bilateral Shoulder Arthroplasty,” Anaesthesiology, Vol. 96, No. 3, 2002, pp. 762-764.

[13] N. S. Sandhu, J. S. Manne, P. Medabalmi and L. M. Capan, "Sonographically Guided Infraclavicular Brachial Plexus Block in Adults: A Retrospective Analysis of 1146 Cases,” Journal of Ultrasound Medicine, Vol. 25, No. 12 2006, pp. 1555-1561.

doi:10.1097/00000542-200203000-00037 